\title{
ENERGY DISSIPATION IN WAVES BREAKING ON GENTLE SLOPES
}

\author{
M.J.F. STIVE
}

Harbours and Coasts Branch, Delft Hydraulics Laboratory, Voorsterweg 28, 8316 PT Marknesse (The Netherlands)

(Received August 2, 1982; revised and accepted October 10, 1983)

\begin{abstract}
Stive, M.J.F., 1984. Energy dissipation in waves breaking on gentle slopes, Coastal Eng., 8: $99-127$.

The flow field of waves breaking on a gently sloping beach is shown to closely resemble that of hydraulic jumps. This supports the use of the hydraulic jump formulation for the breaking wave energy dissipation. A correction to this formulation, which takes into account the effects of turbulent flow, is found to explain the observed discrepancies between the classical theoretical result and the experiments satisfactorily. These findings are used to propose a simple, semi-empirical model for the wave height decay which includes the set-up. The model is generalized to a wider range of wave conditions by analyzing published data.
\end{abstract}

\section{INTRODUCTION}

A main feature of the surfzone is the wave height decay due to the turbulence generated by breaking. Models for the prediction of this decay are of practical importance in coastal engineering. Realistic results may be expected from models which introduce a dissipation function for the turbulent motion in the averaged energy equation.

A formulation of the breaking wave dissipation after that in hydraulic jumps is frequently used (originally by LeMéhauté, 1962; extended by Divoky et al., 1970; Hwang and Divoky, 1972; and many others) based on the visual resemblance between both phenomena. In this paper the assumption of similarity between the two types of flow is investigated by a detailed comparison between the measured flow fields of a hydraulic jump and a quasi-steady breaking wave on a gently sloping beach. These results are used to investigate the modelling of energy dissipation in breaking waves.

The paper is made up as follows. First, a short description of the experiments is given. Secondly, the mean flow and turbulence characteristics in quasi-steady breaking waves are compared to those in a hydraulic jump. Thirdly, with the aid of the measured energy flux the energy dissipation formulated after the hydraulic jump is investigated. Fourthly, a simple 
model for the wave height decay is constructed. Finally, this model is generalized to a wider range of conditions.

This paper is a sequel to two earlier papers studying the two-dimensional breaking of waves on gentle slopes based on the same measurements, i.e. Stive (1980) elaborating the instantaneous velocity and pressure fields and Stive and Wind (1982) elaborating the time-averaged momentum balance.

\section{EXPERIMENTS}

The measurements from which the present results were derived are the same as those used in the two previously published studies (Stive, 1980; Stive and Wind, 1982). To prevent duplication of the description of details just a brief account of the arrangements, instruments and procedures is given below.

\section{Arrangements}

The experiments were conducted in a wave flume of the Delft Hydraulics Laboratory, $55 \mathrm{~m}$ long, $1 \mathrm{~m}$ wide and $1 \mathrm{~m}$ high. Periodic waves with minimal free second-harmonic components were generated in a water depth of $0.85 \mathrm{~m}$. The waves broke on a plane concrete slope 1:40 (see Fig. 1a).

\section{Instrumentation}

Surface elevations in the surfzone were measured by conductivity-type wave gauges positioned $1 \mathrm{~m}$ apart. Although aeration influences the response of the gauges, the air content in the breaking waves was estimated low enough to cause only negligible deviations.

Velocities were measured by means of a laser doppler velocity meter (LDV). The device was mounted on a carriage such that any desired horizontal or vertical position along the flume could be reached. At different levels the horizontal and vertical components of velocity were measured simultaneously with the surface elevation in the cross-sections indicated in Fig. 1b.

\section{Data-analysis}

The velocity data were processed with the aid of an ensemble averaging technique in which each wave cycle in the time series was considered to be one realization. The technique results in a description of the horizontal and vertical velocity components as the sum of an "organized", periodic component (denoted by a tilde) and an "unorganized", residual component (denoted by a prime), i.e. $u=\tilde{u}+u^{\prime}$ and $w=\bar{w}+w^{\prime}$. In the breaking region the residual component is typically formed by the turbulence due to break. 


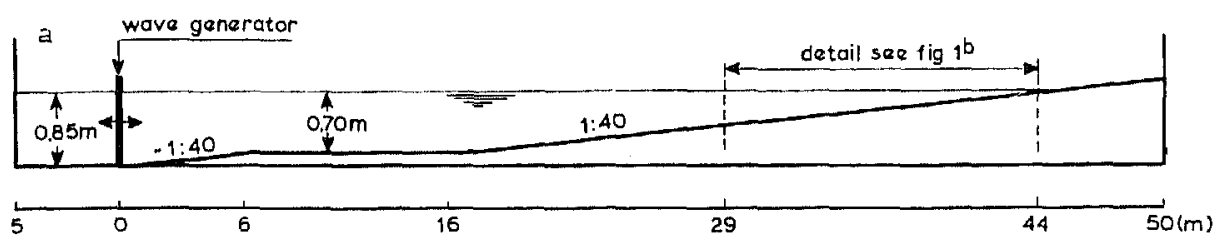

b

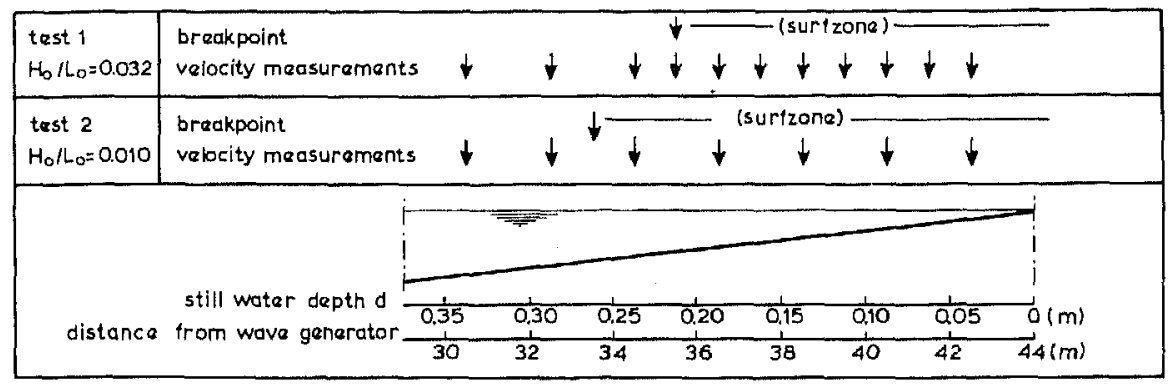

Fig. 1. a. Experimental set-up. b. Detail experimental set-up.

ing. A characteristic order of magnitude of its rms-value is $0.1 c$, where $c$ is the wave propagation speed. In the shoaling region the residual component shows $\mathrm{rms}$-values below $0.01 \mathrm{c}$. The relative low values in the shoaling region confirm the consistency of the ensemble averaging technique.

The presence of air bubbles such as are met with in the crests of breaking waves prevents LDV measurements. However, to derive results for an integral quantity as the energy flux it is necessary to integrate the velocity over the full depth. Therefore, estimates of the velocities in the aerated crest regions were obtained by extrapolation. The method of extrapolation is shortly described and evaluated in Appendix A. Experimental inaccuracies (indicated by error bars in the figures presenting the data) are mainly due to the extrapolation.

\section{Wave conditions}

The experiments were restricted to two conditions (see Table I), which are referred to as test 1 and test 2 . These conditions represent two types of initial breaking usually found on gently sloping beaches. The initial breaking behaviour of test 1 falls in the category spilling breaking, while that of test 2 falls in the category plunging breaking. As characterized by Svendsen et al. (1978), the rapid transitions of wave shape in the region right after breaking - the so-called outer region - develop soon, i.e. after a horizontal distance of several times the breaker depth, into the relatively well-organized, quasi-steady breaking motion of the inner region, which is virtually independent of the initial breaking behaviour. At that stage of their 
breaking motion, breakers on a beach may be described as spilling breakers or bores. Here, the outer region is determined as the region confined between the cross-section where the wave height is maximum (the "breakpoint") and the cross-section where the wave height over water depth ratio is virtually constant.

TABLE I

Wave conditions (wave height $H$, period $T$, wave length $L$, where the subscripts $o, h$ and $b$ denote deep water, horizontal section and breakpoint)

\begin{tabular}{llllll}
\hline Test & $H_{\mathrm{o}}(\mathrm{m})$ & $H_{\mathrm{h}}(\mathrm{m})$ & $H_{\mathrm{b}}(\mathrm{m})$ & $T(\mathrm{~s})$ & $H_{\mathrm{o}} / L_{\mathrm{o}}$ \\
\hline $\mathbf{1}$ & 0.159 & 0.145 & 0.178 & 1.79 & 0.032 \\
2 & 0.142 & 0.145 & 0.226 & 3.00 & 0.010 \\
\hline
\end{tabular}

\section{THE FLOW FIELD SIMILARITY}

The visual resemblance between quasi-steady breaking waves in shallow water and steady bores or hydraulic jumps is frequently mentioned in the literature. A comparison between their mean and turbulent flow characteristics may be made as follows.

Under the assumptions of a (locally) constant wave propagation speed and a (locally) horizontal bottom, breaking wave results acquired in one cross-section may be translated into steady mean flow results by choosing a reference frame which moves with the wave at its local propagation speed $c$. For these steady bores the Froude number $F_{1}$, which indicates the bore strength, corresponds to the celerity at which the uniform flow of the trough meets the surface roller so that:

$F_{1}=\left(c+U_{1 R}\right) /\left(g d_{1 \mathrm{R}}\right)^{1 / 2}$

in which $c$ is the wave propagation speed and $U_{1 \mathrm{R}}$ the depth-averaged velocity at the trough or upstream section, both in the original frame of reference. It is noted that the upstream depth $d_{1 \mathrm{R}}$ is positioned at the initiation point of turbulent flow at the surface (the section indicated $1 \mathrm{R}$ in Fig. 2), which corresponds to the upstream depths commonly taken in hydraulic jump experiments. The Froude numbers thus calculated ranged from 1.4 to 1.6 indicating that quasi-steady breakers belong to the class of weak bores.

A mean flow and a turbulent intensity distribution of a quasi-steady breaker and of a low Froude hydraulic jump (Rouse et al., 1958) are com. pared in Fig. 3. It appears that the flows are similar in the sense that the cross-sectional uniformity of both the mean and the turbulent flow at the toe section is disturbed by the surface roller. The non-uniformity of the 
wake-type flow behind the roller slowly restores to the initial condition of uniformity.

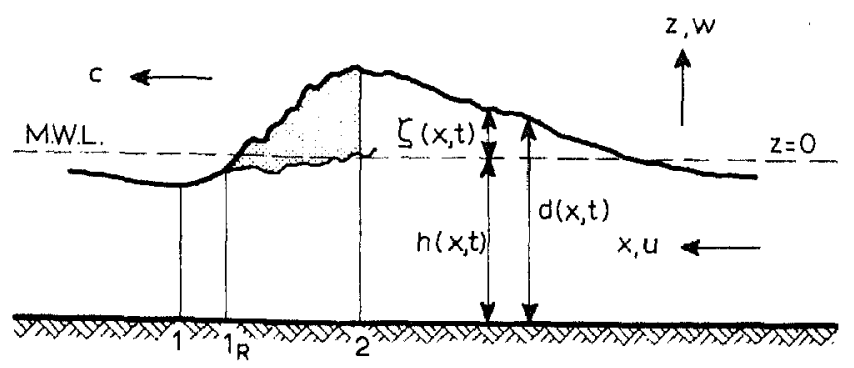

Fig. 2. Quasi-steady breaking wave in a frame of reference stationary with respect to the fixed bottom; coordinates, notation and definitions.

\section{EVALUATION OF WAVE PROPAGATION PROPERTIES}

Key elements in the modelling of the breaking wave energy decay are the flux and the dissipation of wave energy. As elaborated further on this study seeks a simple description for the energy flux and - based upon the similarity in flow - for the energy dissipation a description closely after that in hydraulic jumps. To that end a comparison is made between the measured flux and its linear, shallow-water approximation as well as between the measured dissipation and the hydraulic jump dissipation. An important property in the evaluation of the energy flux is the wave phase speed which is treated first.

\section{Phase speed}

The wave phase speed, $c$, in the breaking region is taken as the mean velocity of propagation of characteristic points of the wave fronts between consecutive measurement cross-sections. In Fig. 4 measurements of $c$ are compared to its linear, shallow-water approximation which reads:

$c=(g h)^{1 / 2}$

It appears that close to and in the outer region the linear, shallow-water approximation underestimates the phase speed $c$. This indicates that nonlinear effects are important, as expected for this region with relatively steep waves. Improvements may be made by introducing non-linear approximations. In the inner region the waves are less steep resulting in somewhat smaller discrepancies between the shallow-water approximation and the measurements. Here significant improvements may be made by introducing the periodic bore approximation, $c_{\text {bore }}$, and accounting for the effects due 
(a) $\longrightarrow \bar{\nabla} / V_{1 R}=(U-c) /\left(U_{1 R}-c\right)$

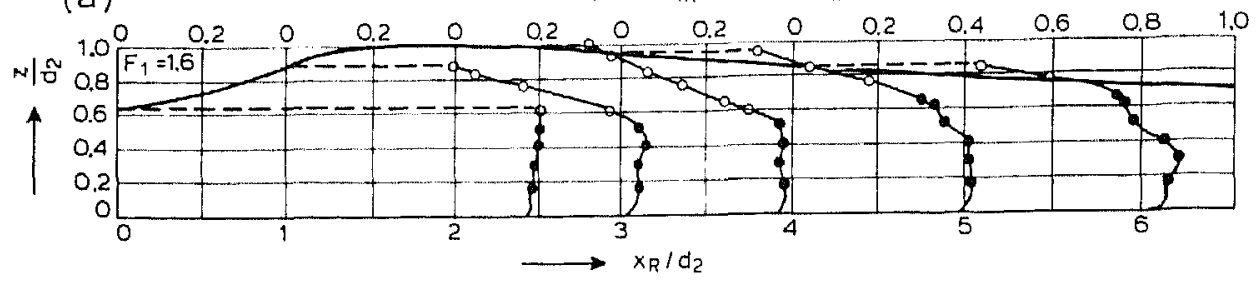

Typical breaking wave result translated to frame with stationary motion (test $1, x=37.5 \mathrm{~m}$ ) - measurements o extrapolations

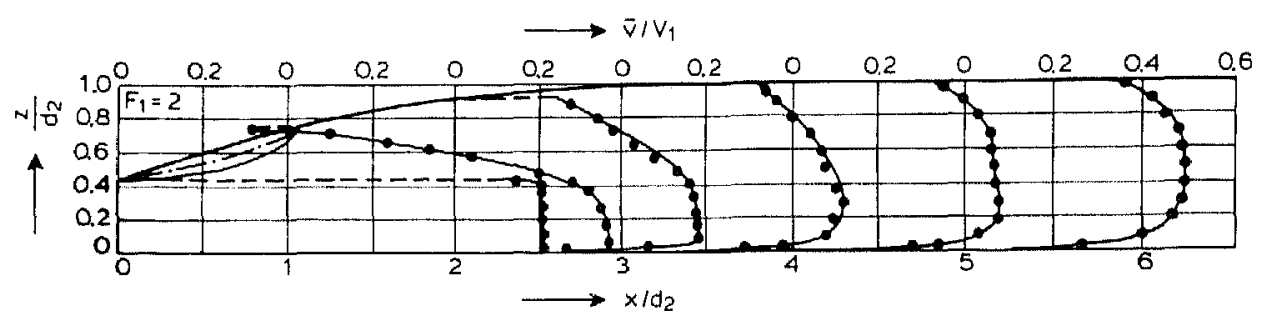

Hydraulic Jump results of Rouse et al (1958)

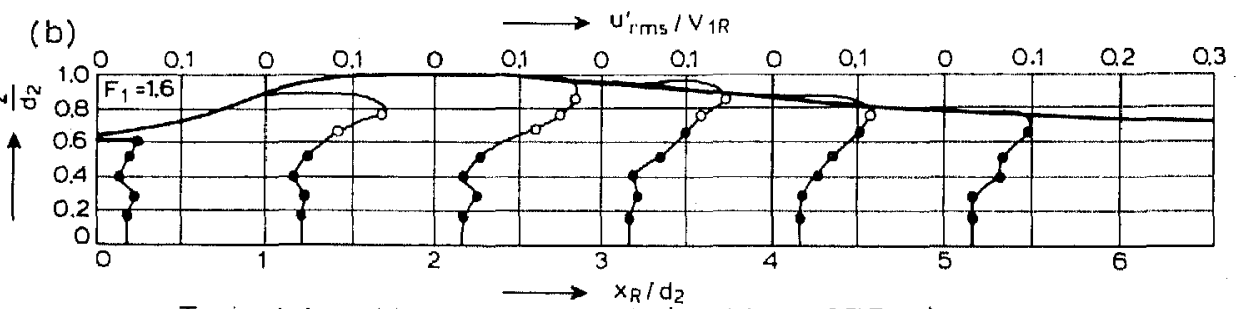

Typical breaking wave result (test $1, x=37.5 \mathrm{~m}$ )

- measurement o extrapolations

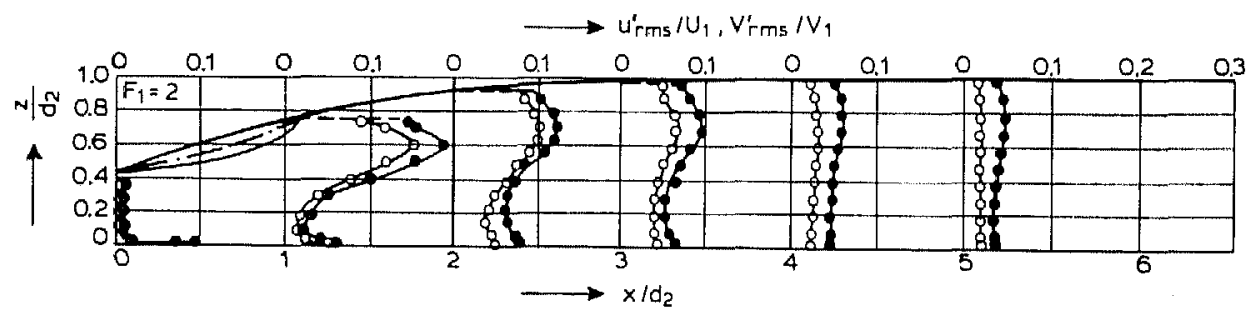

Hydraulic jump results of Rouse et al (1958)

- $w_{r r m s}^{\prime} / V_{1} \cdot u_{r m s}^{r} / V_{1}$

Fig. 3. a. Comparison of mean flow distribution. b. Comparison of turbulent intensity distribution. 

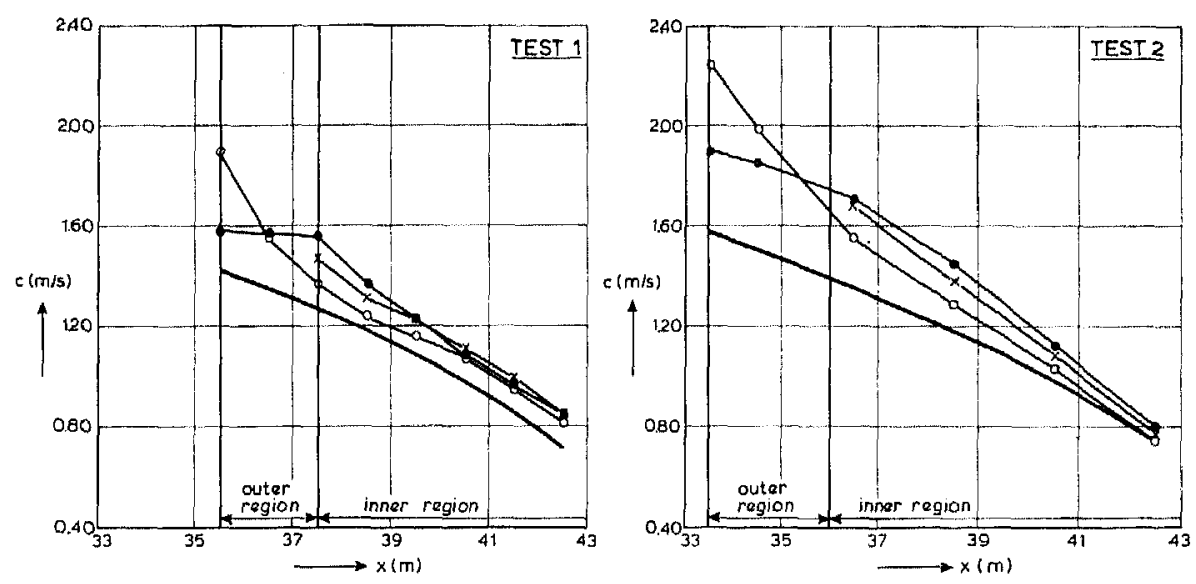

- meosuramants

- 'classical' bore approximation (eq. 4.2)

* 'classical' bora opproximation corracted for turbulant flow effects (eq. 4.2 and eq. b.1)

Fig. 4. Wave phase speed $c$.

to turbulence. These approximations may be derived from an evaluation of the momentum balance equation for a periodic bore (see Svendsen et al., 1978). Neglecting the effects due to turbulence the result is:

$c_{\text {bore }}=\left(1 / 2 g d_{1} d_{2}\left(d_{1}+d_{2}\right) h^{-2}\right)^{1 / 2}$

Turbulent effects may be accounted for in this result by including a correction term $\left(A_{\mathrm{c}}\right)$, which is given in Appendix $\mathrm{B}$.

\section{Energy flux}

The time-averaged or mean energy flux, $\bar{F}$, per unit width is defined as:

$$
\bar{F} \equiv \overline{\int_{d}\left[p+\rho g z+1 / 2 \rho\left(u^{2}+w^{2}\right)\right] u \mathrm{~d} z}
$$

where the integral is performed over the instantaneous fluid depth, $d$, and the overbar denotes time averaging. Furthermore $p$ is the pressure. Based upon the pressure measurements reported in Stive (1983) it was found that the mean energy flux in the quasi-steady breaking region may be approximated to within $10 \%$ by the hydrostatic equivalent of eq. (4.2):

$\bar{F}=\rho g c \overline{\xi(\xi-\bar{\zeta})}+\int_{d} \overline{1 / 2 \rho\left(u^{2}+w^{2}\right) u \mathrm{~d} z}$

The first term is known from surface elevation measurements and the second term from the measured and extrapolated velocity field. Results are given in Fig. 5. In the initial breaking region, the hydrostatic approxima- 
tion is not valid and the extrapolation of the velocities is not possible. It was assumed instead that the mean energy flux varies linearly from the breakpoint to the seaward boundary of the inner region. The mean energy flux at the breakpoint was calculated from the deep water conditions assuming constancy of energy flux up to the breakpoint. Taking into account dissipative effects due to the channel friction the latter estimate may also be assumed accurate to within $10 \%$.
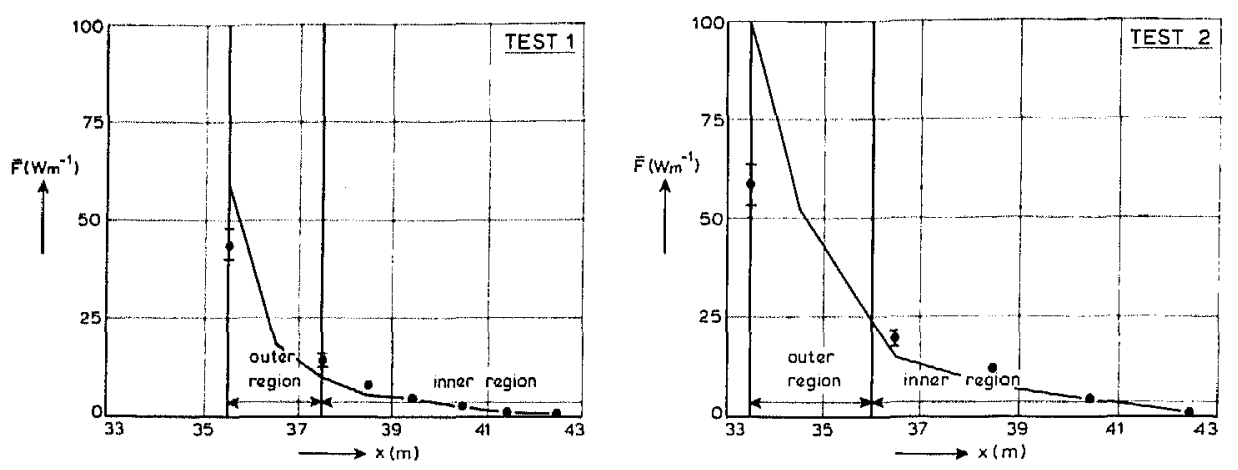

- measurements

Fig. 5, Mean energy flux $\bar{F}$.

The linear, shallow-water approximation to the mean energy flux reads:

$\bar{F}=1 / 8 \rho H^{2}(g h)^{1 / 2}$

This approximation is compared to the measurements in Fig. 5. The observed discrepancies in the outer region are again due to non-linear effects. These effects decrease significantly in the inner region resulting in only small discrepancies between the linear, shallow-water approximation and the measurements.

\section{Energy dissipation}

The measured energy dissipation is derived from the measured spatial variation of the mean energy flux through application of the mean energy balance for a control volume which is stationary with respect to the fixed bottom and which extends vertically from the bottom to the free surface and horizontally from one measurement cross-section to another over a distance $\Delta x$. So the measured mean dissipation rate per unit area, $\bar{\epsilon}_{\text {meas, }}$ is given by:

$\bar{\epsilon}_{\text {meas. }}=-\Delta \bar{F}_{\text {meas. }} / \Delta x$

A comparison between the measured dissipation and the dissipation formulated after the hydraulic jump may be made as follows. As described in 
Appendix B the result for the dissipation rate of a periodic jump, which may be considered as the periodic version of the classical result, reads:

$\epsilon_{\text {bore }}=\rho g c_{\text {bore }} h\left(d_{2}-d_{1}\right)^{3} /\left(4 d_{1} d_{2}\right)$

After conversion of this result to an average dissipation rate per unit area through dividing by the wave length $L$ and after equating the height of the jump to the wave height, i.e. $d_{2}-d_{1}=H$, the average breaking wave dissipation rate per unit area formulated after the hydraulic jump may be expressed as:

$\bar{\epsilon}=\bar{\epsilon}_{\text {bore }} A_{\epsilon}$

where $\bar{\epsilon}_{\text {bore }}=\epsilon_{\text {bore }} / L=1 / 4 \rho g c H^{3} h /\left(d_{1} d_{2} L\right)$

and where the non-dimensional dissipation factor $A_{\epsilon}$ accounts for the differences between the dissipation in a bore and that in a classical hydraulic jump, for which $A_{\epsilon}$ equals 1 . From the measured variations of energy flux, wave height and wave propagation speed a measured value for $A_{\varepsilon}$ was calculated by combining eqs. (4.4) and (4.6):

$A_{e_{\text {meas. }}}=\left(-\Delta \bar{F}_{\text {meas. }} / \Delta x\right) / \bar{\epsilon}_{\text {bore }}$

where $\Delta \bar{F}_{\text {meas. }}$ is the change in energy flux measured in consecutive crosssections at a distance of $\Delta x$. This indirect representation of the measured dissipation facilitates the comparison relative to the classical hydraulic jump formulation.

The results for the dissipation factor $A_{e}$ shown in Fig. 6 indicate that in the inner breaking region the classical jump result underestimates the measured dissipation by 30 to $50 \%$ depending mainly on the deep-water wave steepness and less on the distance to the initial breaking region. In the outer breaking region the agreement is closer. It must, however, be questioned whether this is a matter of coincidence since the highly unstationary, jetlike motion can hardly be expected to be described by the bore motion. Therefore no closer investigation will be made for this region.

Regarding the inner breaking region improvements on the hydraulic jump dissipation according to expression (4.5) may be attempted by introducing formal corrections for the assumptions made in the classical case. The classical assumptions that apply to both the upstream (trough) and downstream (crest) section are: (1) the flow is free of turbulence; (2) the mean horizontal velocity is uniform over the depth; and (3) the pressure distribution is hydrostatic.

The effect of deviations from these assumptions may be investigated by introducing correction coefficients, i.e. a coefficient $\alpha$ in the momentum equation and a coefficient $\beta$ in the energy equation which both equal 1 in the classical case. The definitions of $\alpha$ and $\beta$ introduced to express the corrections are given in Table II. Deviations from the classical assumptions 


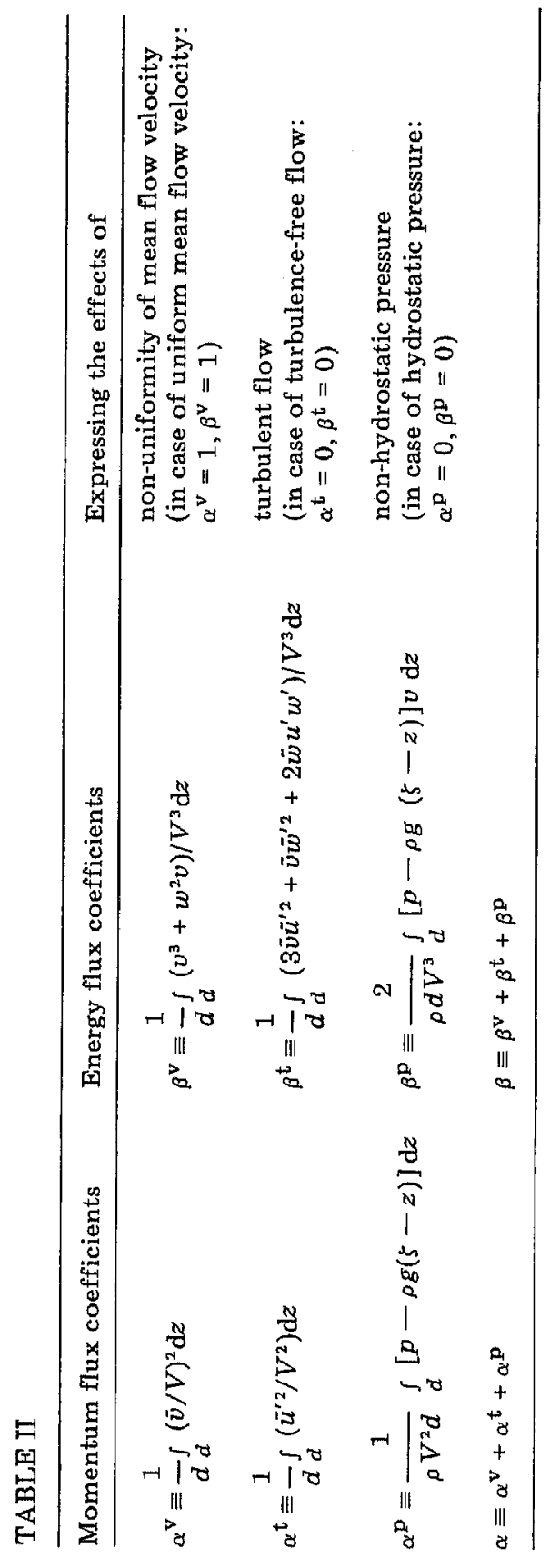



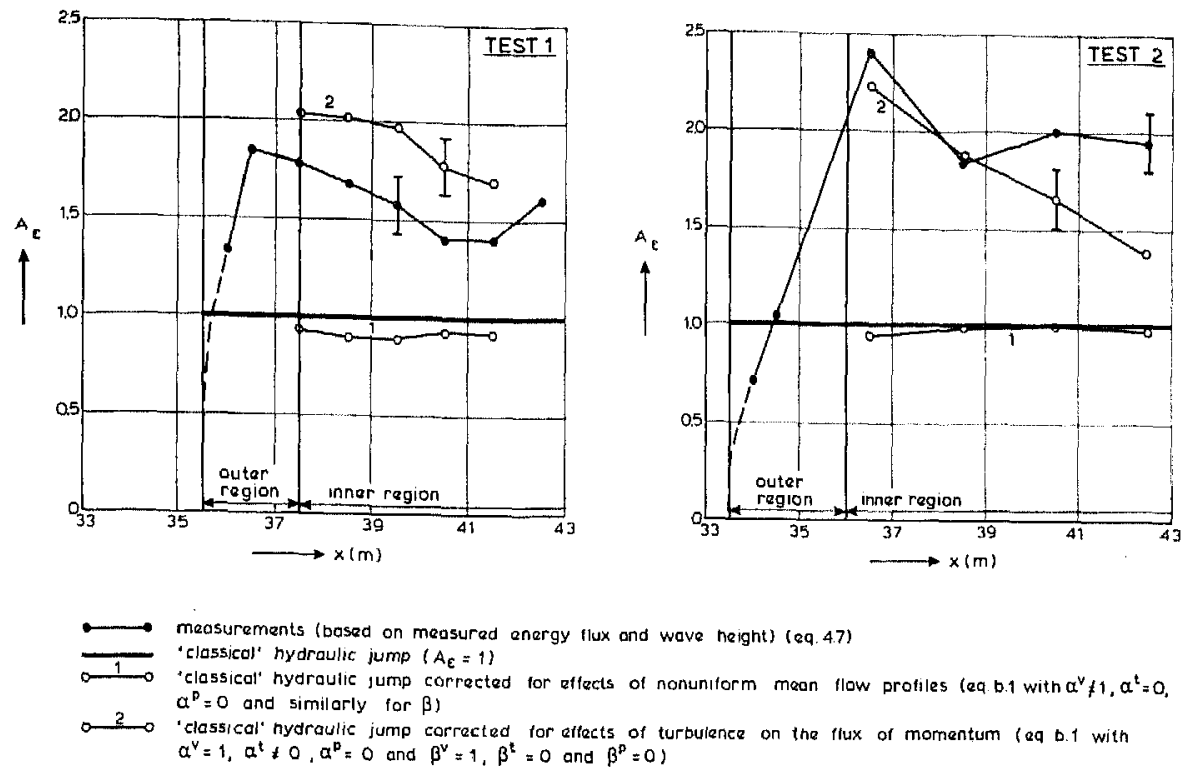

Fig. 6. Non-dimensional dissipation $A_{e}$.

are accounted for by deviations of $\alpha$ and $\beta$ from 1 , which result in corrections to $\epsilon_{\text {bore }}$ according to expression (b.1) as derived in Appendix B.

It is noted that in defining the coefficients the horizontal ensemble mean velocities are transformed to $\tilde{v}=\tilde{u}-c$ so that the ensemble mean motion is steady. Furthermore, a depth-averaged horizontal velocity is introduced as:

$V \equiv \frac{1}{d} \int_{d} \bar{v} \mathrm{~d} z$

The coefficients $\alpha^{\mathrm{v}}, \beta^{\mathrm{v}}, \alpha^{\mathrm{t}}$ and $\beta^{\mathrm{t}}$ could be calculated directly from the measured internal flow fields. In the upstream section 1 the coefficients are very close to 1 and 0 implying nearly depth-uniform, turbulence-free flow conditions. The results for section 2 are given in Fig. 7, where they are compared to the values as calculated from the measurements of Rouse et al. (1958) in their cross-sections 2 and 3 , which are estimated to be equivalent to the crest section of the breakers from inspection of Fig. a.2.

It appears that the non-uniformity coefficients $\alpha^{\mathrm{v}}$ and $\beta^{\mathrm{v}}$ are comparable in breakers and in jumps, but that the turbulence coefficients $\alpha^{t}$ and in particular $\beta^{t}$ are somewhat lower in breakers than in jumps, which is probably due to the weaker bore strength of the quasi-steady breakers.

The measured coefficients were applied to account for their effects on the estimated dissipation (relative to the classical hydraulic jump formulation) through equation (b.1) as given in Appendix B. These effects are il- 


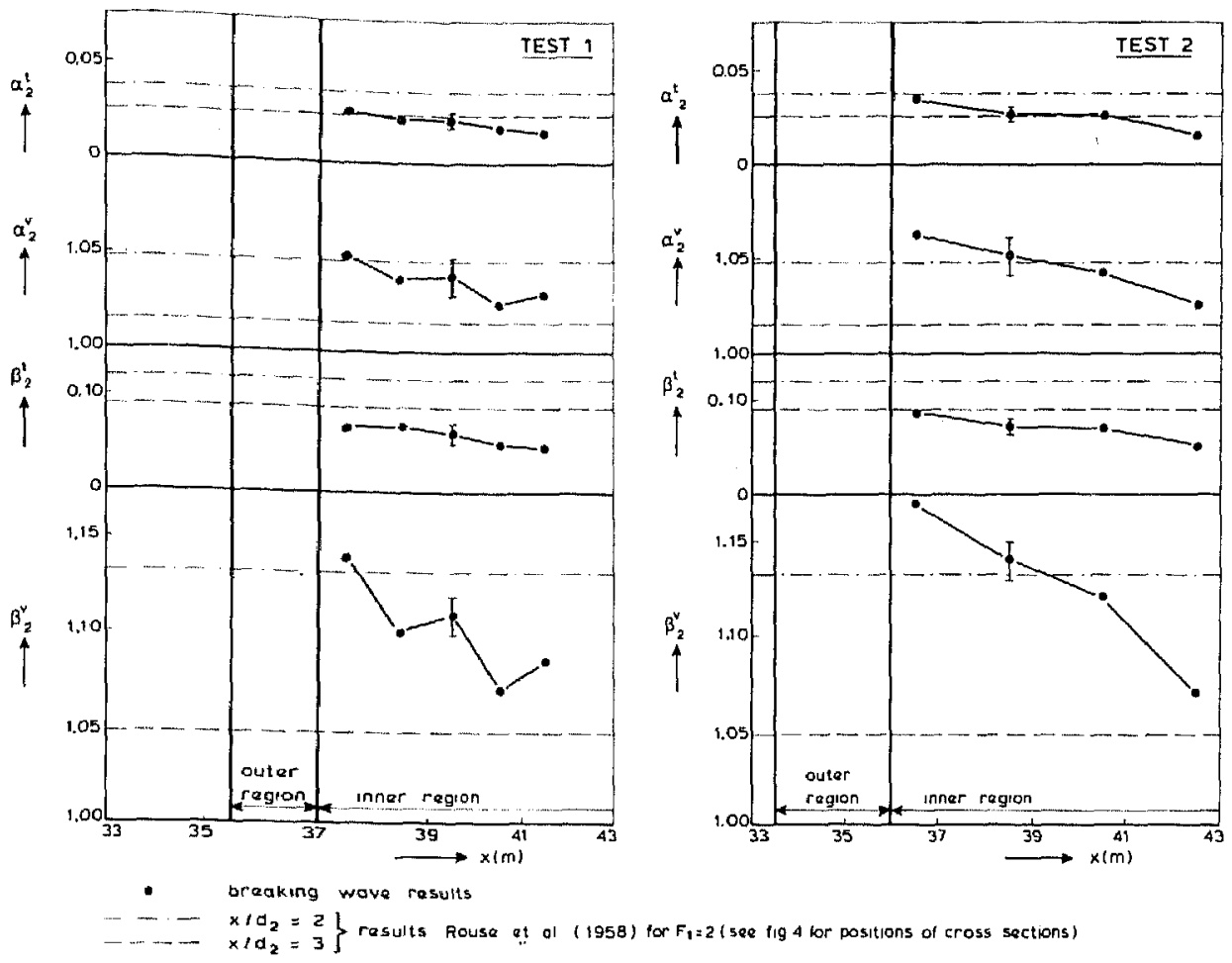

Fig. 7. Coefficients expressing the effects of non-uniform mean flow profiles and of turbulent flow.

lustrated by the curves 1 and 2 in Fig. 6. Curve 1 only incorporates the effects of non-uniformity of the flow profile $\left(\alpha^{v} \neq 1, \beta^{v} \neq 1\right)$ at the crest. Curve 2 only incorporates the effects of turbulence at the crest so far as it concerns the flux of momentum $\left(\alpha^{t} \neq 0\right)$. Here the effects of turbulence on the flux of energy are neglected (i.e. $\beta^{t}=0$ ) based on the consideration that the turbulent energy convected downstream through section 2 will dissipate after all. From the results in Fig. 6 it may be concluded that the major correction to the estimated jump dissipation is due to the turbulent flux of momentum at the crest section. This correction increases the estimated jump dissipation such that it only differs a small amount from the measured dissipation.

The effects of non-hydrostatic pressure could not be derived directly from the measurements. Estimates were made based on the approach described in Svendsen and Madsen (1981) who use the curvature of the streamlines in the nearly steady waves to derive the effects of non-hydrostatic pressure. This approach agreed well with the analysis of pressure results described in Stive (1983). Based on the measured surface curvatures and the measured mean flow profiles, the estimated jump dissipation was calculated to increase $10 \%$ maximally. 
An additional effect increasing the jump dissipation but not yet accounted for is the production and dissipation of turbulence behind the crest section. This turbulence is produced by the shear due to the non-uniform flow profiles behind the crest section. From the energy balance made up by Rouse et al. (1958) for their weakest jump it may however be concluded that the production and dissipation behind the downstream section equivalent to the present crest section is less than $10 \%$ of the magnitude of the estimated jump dissipation.

Summarizing it may be concluded that the dissipation rate of the presently investigated quasi-steady breaking waves is underestimated by the classical hydraulic jump approach by 30 to $50 \%$. The differences are found to be mainly explained from the effects of turbulent flux of momentum.

\section{A WAVE HEIGHT DECAY MODEL}

\section{The energy balance equation}

Starting point in the derivation of a simple model for the wave height decay is the energy balance equation (4.4) in differential form:

$$
\frac{\mathrm{d}}{\mathrm{d} x} \bar{F}=-\bar{\epsilon}
$$

Since it appears further on that the solution greatly simplifies by introducing the linear, shallow-water approximation for the mean energy flux $\bar{F}$ a definition for $\bar{F}$ is introduced in close analogy to linear, shallow-water wave theory:

$\bar{F} \equiv A_{\mathrm{F}} \rho g H^{2} c$

where $A_{\mathrm{F}}$ is a non-dimensional energy flux.

Substitution of this definition in the energy balance equation yields a still general, first-order differential equation in $H$ :

$\frac{\mathrm{d} H}{\mathrm{~d} x}+\frac{H}{2 c} \frac{\mathrm{d} c}{\mathrm{~d} x}+\frac{H}{2 A_{\mathrm{F}}} \frac{\mathrm{d} A_{\mathrm{F}}}{\mathrm{d} x}=\frac{-\bar{\epsilon}}{2 g H A_{\mathrm{F}} c}$

A key element in solving this equation for $H$ is the energy dissipation $\bar{\epsilon}$. In its turn the dimensionless dissipation factor $A_{\epsilon}$ was shown to be the determining factor for $\bar{\epsilon}$. It is argued here that discrimination between the inner and outer breaking region with respect to the dissipation cannot realistically be made without having obtained more systematical knowledge of the dissipation than presently available. Therefore, a sophisticated approach to the dimensionless dissipation $A_{\epsilon}$ in the sense of $A_{\epsilon}$ being a function of the horizontal position in the surfzone is not justified. Consistent with this it is assumed that the factor $A_{\epsilon}$ is a constant over the surfzone 
on a beach of constant slope, which is at most a function of the deep water wave steepness and perhaps the bottom slope.

Solving the above differential equation (5.1) for $H$ also requires information about $A_{\mathrm{F}}$ and $c$, i.e. the non-dimensional energy flux and the wave propagation speed. An important question now is how well these coefficients have to be approximated to give an acceptable solution for $H$. Clearly the approximations to $A_{F}$ and $c$ will only have to be as accurate as is justified by the approximation to $A_{\epsilon}$, i.e. $A_{E}$ is a constant over the surfzone. This may be investigated by comparing the measured variation of $A_{\varepsilon}$ according to expression (4.7) where $A_{\epsilon}$ is determined from measured values of $F, c$ and $H$, with the variation of $A_{e}$ as derived from approximations to $A_{\mathrm{F}}$ and $c$ and measured values of $H$. Here this is done for the linear shallow water approximations to $A_{\mathrm{F}}$ and $c$, i.e. $A_{\mathrm{F}}=1 / 8$ and $c=(g h)^{1 / 2}$. The results (see Fig. 8 ) show that the applied approximations yield no larger deviations in $A_{\epsilon}$ from a constant value than the measurements. It may therefore be concluded that more accurate approximations to $A_{F}$ and $c$ will not result in more accurate solutions for $H$, so it is sufficient for the present purpose to rely on the linear, shallow-water approximations for $A_{\mathrm{F}}$ and $c$.
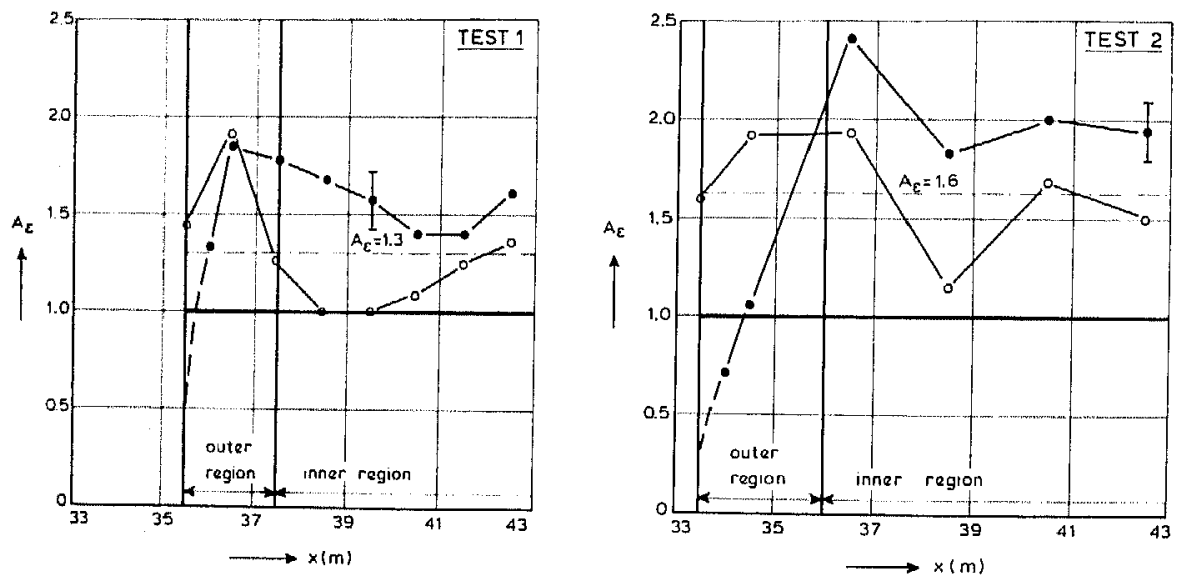

-D- measurements based on measured anergy flux and wave height (eq 4.7)

- measurements based on measured wave height and linear, shallow water approximation to the energy flux
and wave phase speed

Fig. 8. Approximations to the non-dimensional dissipation $A_{\epsilon}$.

Introducing these approximations in the differential equation for $H$
elds: yields:

$\frac{\mathrm{d} H}{\mathrm{~d} x}+\frac{H}{4 h} \frac{\mathrm{d} h}{\mathrm{~d} x}+A_{\epsilon}\left(\frac{h}{g T^{2}}\right)^{1 / 2}\left(\frac{H}{h}\right)^{2}=0$ 
where for $\bar{\epsilon}$ the jump expression (4.4) is used, in which $h / d_{1} d_{2}$ is replaced by $1 / h$ in line with the approximations made so far.

\section{The momentum balance equation}

In the resulting energy balance equation (5.2) we are still left with an unknown term, the mean water depth $h$ which includes the wave set-up $\bar{\zeta}$. Therefore, the averaged momentum equation is introduced to provide a solution for $\bar{\zeta}$ :

$\frac{d S_{x x}}{d x}+\rho g h \frac{d \zeta}{d x}=0$

where $S_{x x}=\overline{\int_{d}\left(p+\rho u^{2}\right)} \mathrm{d} z-1 / 2 \rho g \overline{(\zeta-\bar{\zeta})^{2}}$ is the radiation stress. As was done for the energy flux a definition is introduced in close analogy to linear, shallow water wave theory, i.e.

$S_{x x} \equiv A_{\mathrm{s}} \rho g H^{2}$

where $A_{\mathrm{s}}$ is a non-dimensional radiation stress. The measured variations in $A_{\mathrm{s}}$ (see Stive and Wind, 1982, for details) are given in Fig. 9 where they are compared to the linear, shallow-water approximation $A_{s}=3 / 16$. The discrepancies are most pronounced in the outer breaking region, again indicating that in this region non-linear effects are important. Consistent with the foregoing approximations for the non-dimensional flux and dissipation of energy, the non-dimensional radiation stress $A_{\mathrm{s}}$ is assumed to be a constant over the surfzone. However, as shown in Stive and Wind (1982), the set-up is ill-predicted unless the effect of non-linearity at the breakpoint is taken into account. Therefore $A_{\mathrm{s}}$ is allowed to deviate from the linear, shallow-water approximation $\left(A_{\mathrm{s}}=3 / 16\right)$ in order to reproduce a mean set-up
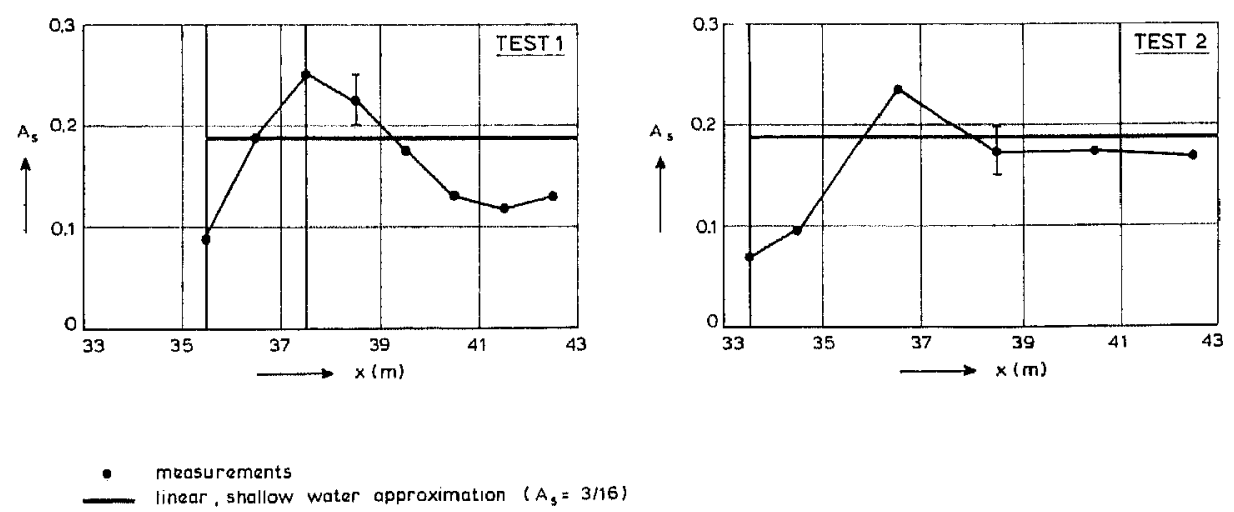

Fig. 9. Non-dimensional radiation stress $A_{\mathrm{s}}$. 
slope of the right order of magnitude, without abandoning the assumption that $A_{\mathrm{s}}$ is constant over the surfzone. After some rewriting the following additional differential equation now arises:

$\frac{\mathrm{d} H}{\mathrm{~d} x}+\frac{h}{2 A_{\mathrm{s}} H} \frac{\mathrm{d} h}{\mathrm{~d} x}+\frac{h}{2 A_{\mathrm{s}} H} s_{\mathrm{o}}=0$

where $s_{\mathrm{o}}=-\mathrm{d} h_{\mathrm{SWL}} / \mathrm{d} x$ is the bottom slope.

\section{Solutions}

The pair of first-order, non-linear differential equations, equations (5.2) and (5.3), may be solved numerically given a value for the factors $A_{e}$ and $A_{\mathrm{s}}$ and initial values for $H$ and $h$ at for instance the point of initial breaking. A second-order Runge-Kutta procedure was used in the numerical evaluation of the differential equations. A step length of the order of the water depth at the breakpoint already proved to yield stable numerical results. However, close to the waterline where the ratio $H / h$ increases rapidly the results explode. This phenomenon is closely related to the classical shoreline singularity for dimensionless progressive waves.

An analytical solution to the problem may be found by assuming the set-up slope to be linearly proportional to the beach slope $s_{0}$. In this simplified case equation (5.3) becomes superfluous. For the present experiments with a beach of constant slope, this implies that the gradient of the mean water depth, i.e. $\mathrm{d} h / \mathrm{d} x=m-s_{\mathrm{O}}=-s$, is also a constant. After normalization of the wave height and mean water depth by their breakpoint values, i.e. $\hat{H}=H / H_{\mathrm{b}}$ and $\hat{h}=h / h_{\mathrm{b}}$, equation (5.2) may be rearranged to yield:

$\frac{\mathrm{d} \hat{H}}{\mathrm{~d} \hat{h}}+\frac{\hat{H}}{4 \hat{h}}-\sigma \frac{\hat{H}^{2}}{\hat{h}^{3 / 2}}=0$

where

$\sigma=\frac{A_{\epsilon}}{s}\left(\frac{H_{\mathrm{b}}}{h_{\mathrm{b}}}\right)\left(\frac{h_{\mathrm{b}}}{g T^{2}}\right)^{1 / 2}$

which is a constant for a given wave steepness and beach slope. The solution to (5.4), under the initial condition $\hat{H}=1$ for $\hat{h}=1$, is given by:

$\hat{H}^{-1}=\left(1-\frac{4}{3} \sigma\right) \hat{h}^{1 / 4}+\frac{4}{3} \sigma \hat{h}^{-1 / 2}$

Note that $\hat{H} \rightarrow\left(\frac{4}{3} \sigma\right)^{-1} \hat{h}^{1, j}$ for $\hat{h} \rightarrow 0$ so that this model predicts a vanishing wave height at the water line but like the numerical model a diverging $H / h$ ratio. Physical validity of both models close to the water line may be enhanced by incorporating additional physical damping mechanisms. 
An advantage of the analytical solution is that the sensitivity of the wave height decay to variations in $\sigma$ and thus in the dissipation factor $A_{\epsilon}$ is easily inferred. Values of $\hat{H}$ and of $\hat{H} / \hat{h}$ versus $\hat{h}$, according to equation (5.6), are presented in Fig. 10 for a range of $\sigma$ values. This range is further on shown to be applicable to the considered wave conditions.
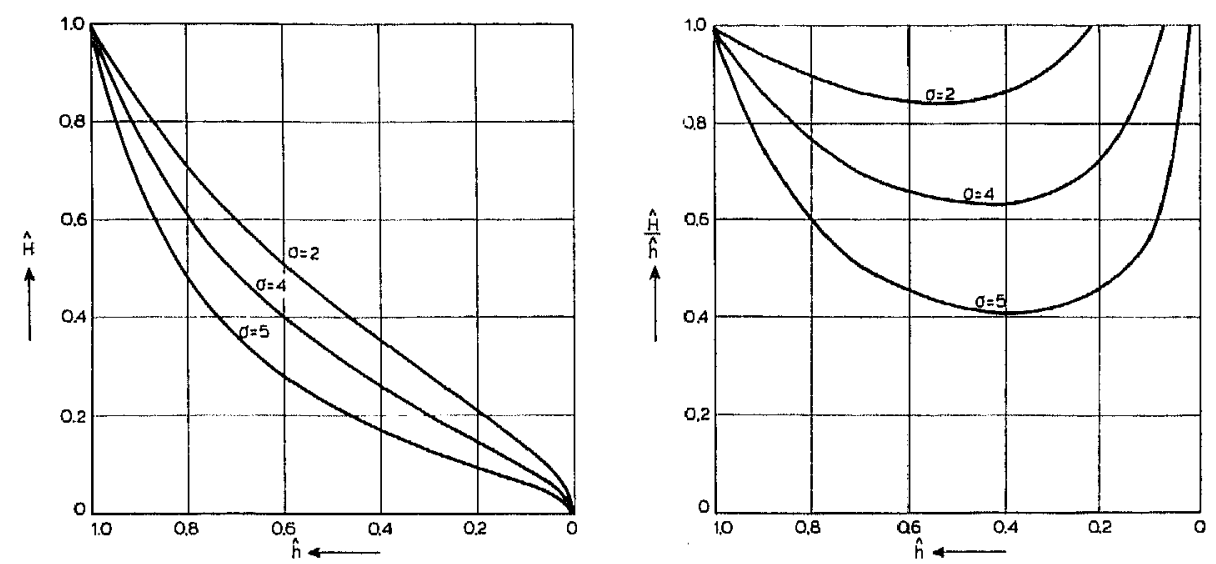

Fig. 10. Results of analytical model; normalized wave height and wave height to mean water depth ratio versus mean water depth.

\section{Comparison of measurements and computations}

A comparison between the numerical and analytical model results and the experimental results for both tests is presented in Fig. 11. In the model calculations, the constant dissipation factor $A_{e}$ was set equal to the average over the surfzone of $A_{\epsilon}$ as calculated from the linear, shallow-water approximation to $A_{\mathrm{F}}$ and $c$ and the measured wave heights, i.e. $A_{\epsilon}=1.3$ for test 1 and $A_{\epsilon}=1.6$ for test 2 (see Fig. 8). The value applied in the numerical model for the radiation stress factor $A_{\mathrm{s}}$ was taken as the value calculated to yield the measured surfzone averaged set-up slope, i.e. $A_{\mathrm{s}}=0.11$ for test 1 and $A_{\mathrm{s}}=0.10$ for test 2 . In the analytical model the mean slope of the total water depth (which is essentially a constant) was taken equal to the one measured.

The results for the computed normalized wave height versus mean water depth of both the numerical and the analytical model are in very satisfactory agreement with the experimental results (Fig. 11). The numerical results for the normalized set-up, i.e. $\bar{\xi}=\bar{\xi} / h_{\mathrm{b}}$, versus mean water depth are less satisfactory. Because of the empirical correction to the radiation stress factor the mean set-up slope is well reproduced, of course. However, the qualitative variation of the set-up is only partially predicted. Specifically in the outer breaking region the prediction is poor which is due to the effects of non-linearity as discussed in Stive and Wind (1982). 

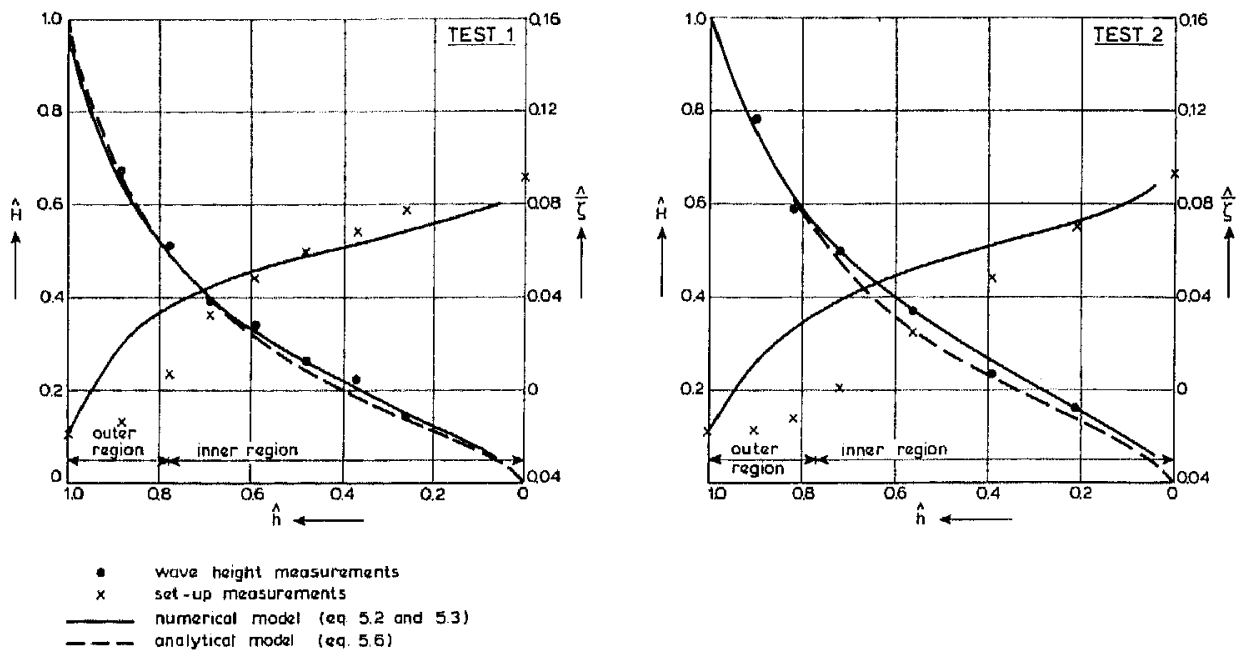

Fig. 11. Comparison between model results and experiments; normalized wave height and set-up versus mean water depth.

The following intermediate conclusions may now be drawn. In view of the fact that the dissipation factor and the radiation stress factor were more or less empirically determined, it is not surprising that the wave height decay and the set-up are reasonably predicted in order of magnitude. A surprising result is, however, that contrary to the set-up the wave height decay is predicted quite accurately in the whole of the surfzone, despite the rather crude modelling of $A_{\epsilon}$ and $A_{\mathrm{s}}$ as constants over the surfzone. Apparently the set-up is a more sensitive quantity in this respect than the wave height.

\section{GENERALIZATION OF THE MODEL}

The relative success of the described model to simulate the wave height decay for the present tests makes it worthwhile to investigate a generalization of the model parameters $A_{\epsilon}$ and $A_{\mathrm{s}}$ for a wider range of wave conditions. To this end published data on wave height decay and set-up were analyzed. The analysis is logically confined to those conditions for which the surfzone has a significant inner breaking region. These conditions are met for waves of moderate steepness on beaches of a slope of 1 in 20 and smaller. In terms of the similarity parameters $\xi_{\mathrm{o}}=s_{\mathrm{o}}\left(H_{\mathrm{o}} / L_{\mathrm{o}}\right)^{-1 / 2}$ or $\xi_{\mathrm{b}}=$ $s_{\mathrm{o}}\left(H_{\mathrm{b}} / L_{\mathrm{o}}\right)^{-1 / 2}$ (Battjes, 1974) this corresponds to $\xi_{\mathrm{o}} \leqslant 0.5$ or $\xi_{\mathrm{b}} \leqslant 0.4$.

The results of this study led to the supposition that the dissipation factor $A_{\varepsilon}$ is a function of the deep-water wave steepness which may be explained as follows. The bore strength was found to vary inversely with the wave steepness. In its turn it is the bore strength which determines the intensity of the turbulent effects at the crest section (see Fig. 7) and thus the mag- 
nitude of the dissipation factor $A_{e}$. Since a decrease in bottom slope may be expected to have qualitatively the same effect on $A_{\epsilon}$ as an increase in incident wave steepness $A_{\epsilon}$ is more generally assumed to be a function of the surf-similarity parameter $\xi_{0}$.

The results of the previous study (Stive and Wind, 1982) led to the supposition that the radiation stress factor $A_{\mathrm{s}}$ is a function of the relative water depth at the breakpoint, $h_{\mathrm{b}} / L_{\mathrm{o}}$. This may be explained by the fact that the set-up is strongly determined by the non-linear effects at and around the breakpoint, which effects will be more pronounced for decreasing relative water depths.

Based on the above assumptions, generalized expressions for the model parameters $A_{\varepsilon}$ and $A_{\mathrm{s}}$ were sought from data of Horikawa and Kuo (1966) on wave height decay and data of Van Dorn (1976) and Singamsetti and Wind (1980) on wave height decay and set-up as follows.

Restriction of the analysis to beach slopes of 1 in 20 and smaller yields a total of eight data sets describing the wave height decay on slopes ranging from 1 in 20 to 1 in 80 (see Fig. $12 \mathrm{a}-\mathrm{h}$, the hatched bands represent the data scatter). For each slope a range of $\sigma$-values was determined (see Fig. 13a) for which the analytically derived wave height decay curves cover the band of scattered wave height data well. In determining $\sigma$ the midpoint value of the given wave steepness range was taken a priori. The scatter in the data does not allow a discrimination between conditions of different wave steepness on a particular slope. The indicated variation in $\sigma$-values is thus incorporating effects of scatter and of a variation in wave steepness. From relation (5.5) the resulting range of values for the dissipation factor $A_{\epsilon}$ could be derived (Fig. 13a). As expected the dissipation factor is an increasing function of the similarity parameter $\xi_{0}$ which may be approximated by:

$A_{\epsilon} \simeq 2 \tanh 5 \xi_{\text {o }}$

Values for $A_{\epsilon}<1$ in the lower range of $\xi_{0}$ are likely to correspond to very weak bores which take up only a fraction of the wave height. Regarding the variation of the analytical model parameter $\sigma$ it is interesting to note that $\sigma$ itself is closely related to $\xi_{\mathrm{b}}$ and thus to $\xi_{\mathrm{o}}$. Relation (5.5) may be rewritten to yield:

$\sigma=\left(\frac{1}{2 \pi} \frac{H_{\mathrm{b}}}{h_{\mathrm{b}}}\right)^{1 / 2} A_{\epsilon} \xi_{\mathrm{b}}^{-1}$

Note that from equation (6.1), $A_{e} \xi_{0}^{-1} \rightarrow 10$ for $\xi_{0} \rightarrow 0$. Since $\xi_{b} \simeq \xi_{0}$ for small $\xi_{0}$ it follows from $(6.2)$ that $\sigma \rightarrow 4\left(H_{\mathrm{b}} / h_{\mathrm{b}}\right)^{1 / 2}$ which explains the constancy of $\sigma$ in the lower range of $\xi_{0}$ (Fig. 13b).

Inspection of the set-up data presented by Van Dorn (1976) and Singamsetti and Wind (1980) indicated that these data are more stable than the 

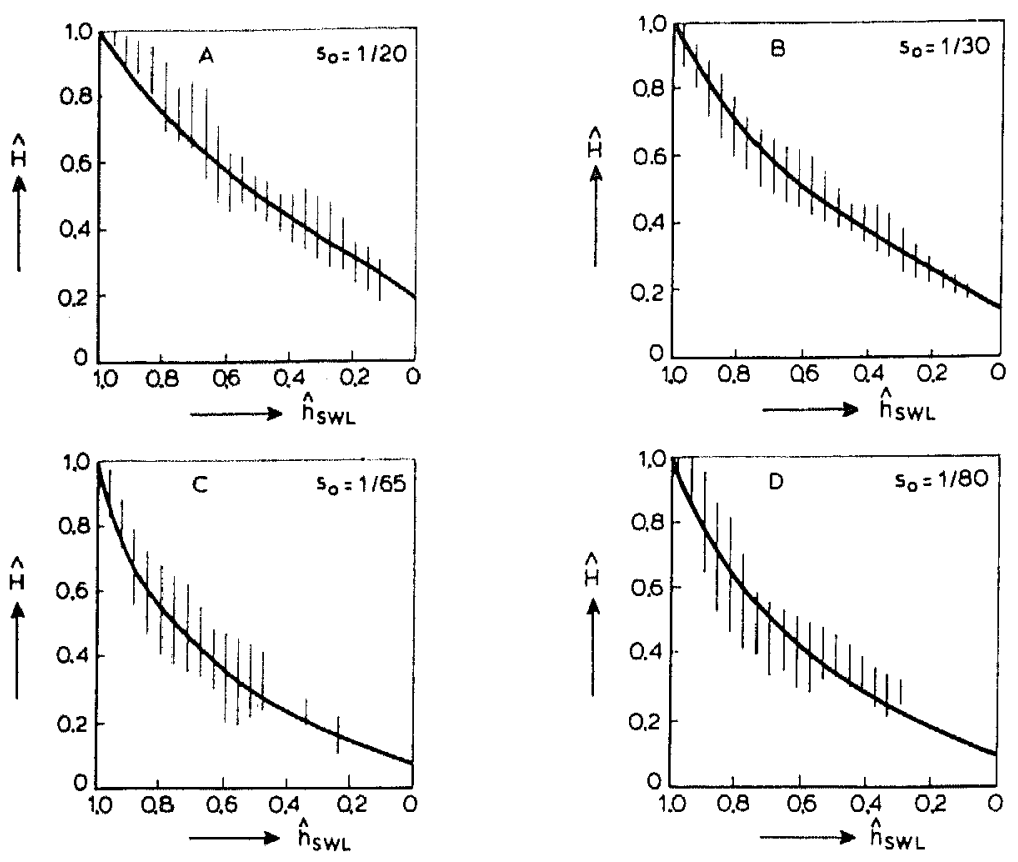

A ....... D Horikawa en Kuo (1966)
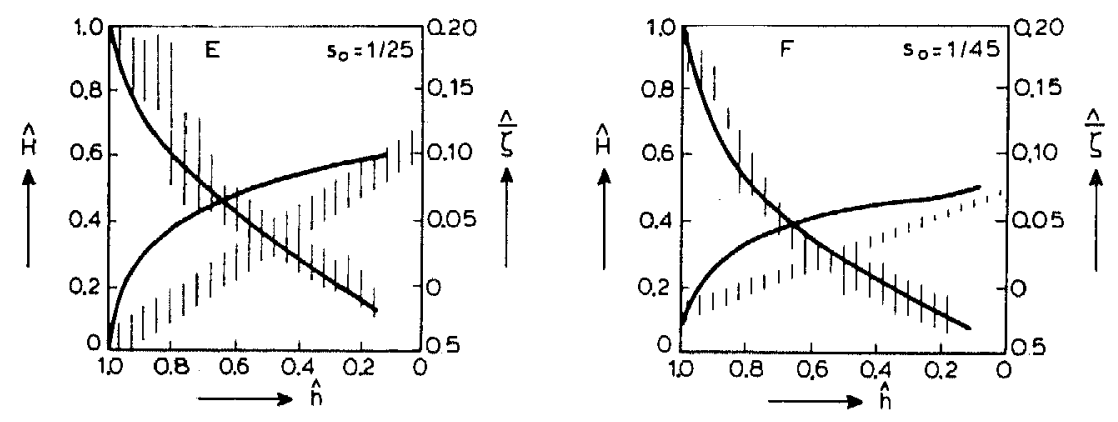

E. F Van Dorn (1976)
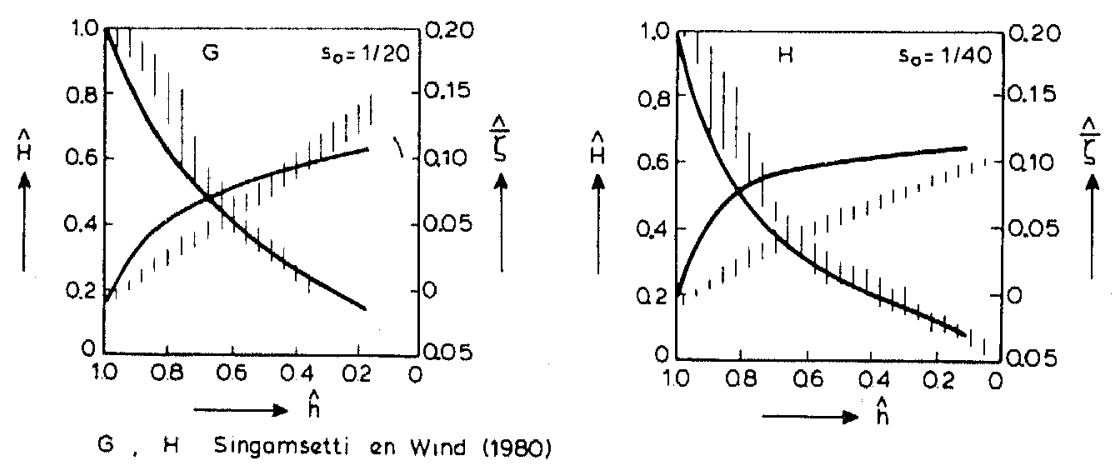

Fig. 12. Comparison of model result with published data; see text for explanation. 

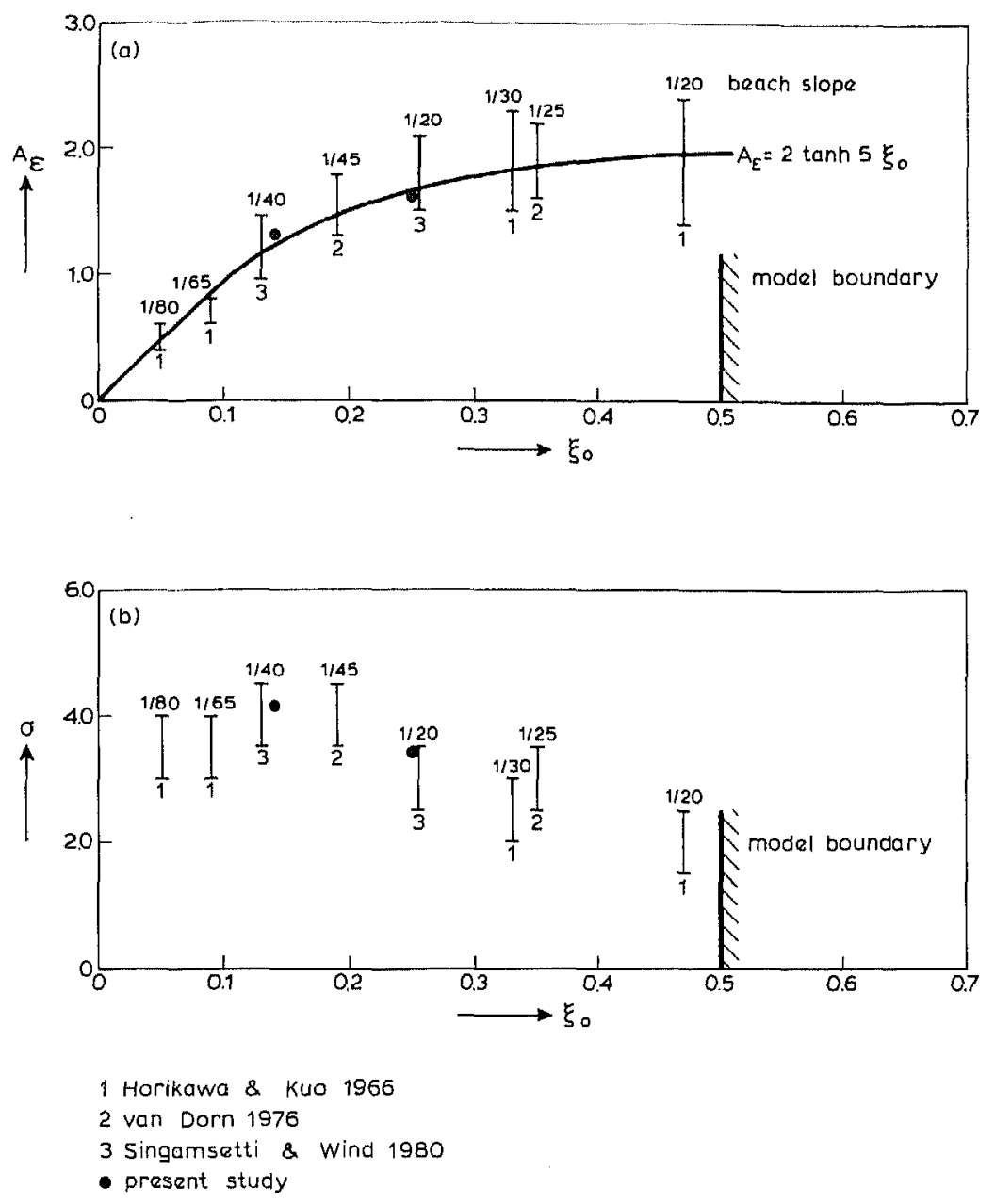

Fig. 13. Non-dimensional dissipation $A_{\epsilon}$ and related factor $\sigma$ as functions of the surfsimilarity parameter $\xi_{0}$.

wave height decay data. Yet the set-up data are also indicated by one hatched band per slope (see Fig. $12 \mathrm{e}-\mathrm{h}$ ), since the data were found to show very little variation with the incident wave steepness after normalization by the water depth at the breakpoint. So in this case the hatched band is incorporating effects of a variation in wave steepness mainly. For each of the set-up measurements a value for the radiation stress factor $A_{\mathrm{s}}$ was determined for which the surfzone averaged set-up slope is reproduced. These values showed no systematic variation with $\xi_{0}$ but as expected they are found to vary systematically with the relative water depth at the break- 
point, $h_{\mathrm{b}} / L_{\mathrm{o}}$ (see Fig. 14). In this figure theoretical breakpoint values for $A_{\mathrm{s}}$ are indicated according to the linear theory, the linear, shallow-water theory and Cokelet's (1976) non-linear theory for waves of maximum energy flux. The correlation between the non-linear theoretical variation and the measured variation of $A_{\mathrm{s}}$ confirms the importance of the nonlinearities at the breakpoint for the set-up. For the present purpose it is sufficient to approximate $A_{s}$ by:

$A_{\mathrm{s}}=0.08+0.88 h_{\mathrm{b}} / L_{\mathrm{o}}$ for $h_{\mathrm{b}} / L_{\mathrm{o}}<0.08$,

while for $h_{\mathrm{b}} / L_{\mathrm{o}} \geqslant 0.08$ (where few data are available) the linear approximation seems to suffice.

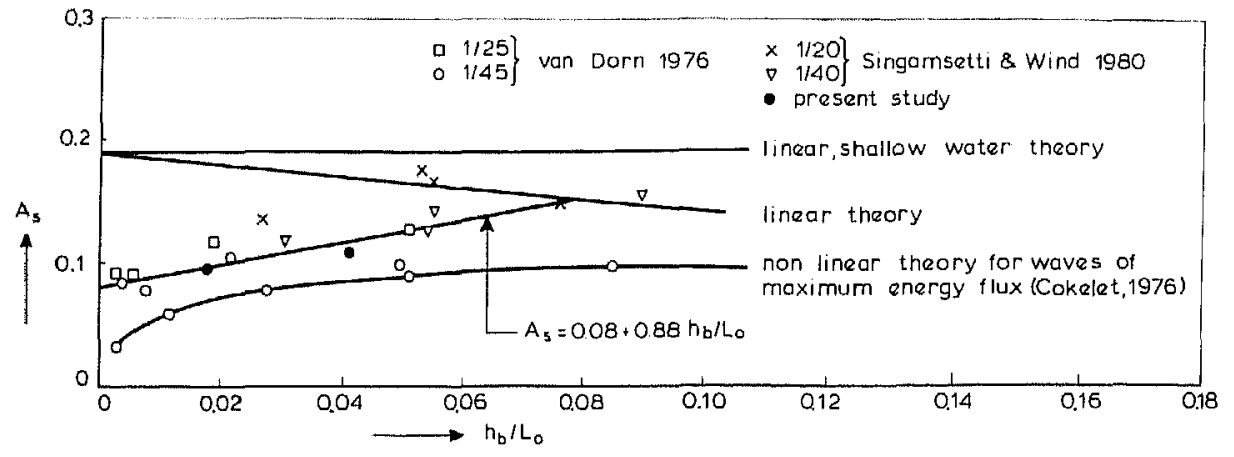

Fig. 14. Non-dimensional radiation stress $A_{s}$ as a function of the relative breaker depth $h_{\mathrm{b}} / L_{\mathrm{o}}$.

Numerical model results for the wave height decay and, where relevant, for the set-up based on the derived expressions (6.1) and (6.3) for $A_{\epsilon}$ and $A_{\mathrm{s}}$ are compared to the published data in Fig. $12 \mathrm{a}-\mathrm{h}$ (for each of the slopes the midpoint wave steepness is taken). Inspection of these figures reveals a satisfactory agreement between calculated and measured wave height decay although the initial decay rate is overestimated in most cases. The agreement between measured and calculated set-up is less satisfactory. As expected the surfzone averaged set-up slope is reasonably predicted, but the set-up variations are qualitatively ill-predicted. The discrepancies are mainly due to a pronounced overestimate of the initial set-up. It is concluded that improved predictions of the wave height decay and specifically the set-up require a more sophisticated modelling of $A_{\epsilon}$ and $A_{\mathrm{s}}$ in the outer breaking region.

Finally, an independent check on the modelling of the wave height decay in the inner breaking region may be obtained from a comparison with more refined data from Svendsen et al. (1978). Like the present experiments their experiments were carried out with periodic waves deprived of their free second harmonics. As a result the scatter in their data is re- 
duced enough to reveal the influence of the incident wave steepness on the wave height decay in the inner breaking region. Here the measured and calculated ratios of wave height to mean water depth are compared for four tests with incident wave steepnesses varying between 0.009 and 0.034 (see Fig. 15). The model calculations are again based on numerical integration of equations (5.2) and (5.3) using the parameter expressions (6.1) and (6.3). It is noted that this is an independent check on the modelling of the dissipation factor as a function of the surf-similarity parameter [equation (6.1)], since this equation was based on variations in bottom slope only. Taking into account that the ratio $H / h$ is a rather sensitive quantity, which is reflected in the still existing data scatter, and that the present model contains insufficient physical damping near the shore line, it may be concluded that the model predicts the influence of the incident wave steepness on the wave height decay in the inner breaking region qualitatively well and quantitatively reasonably.

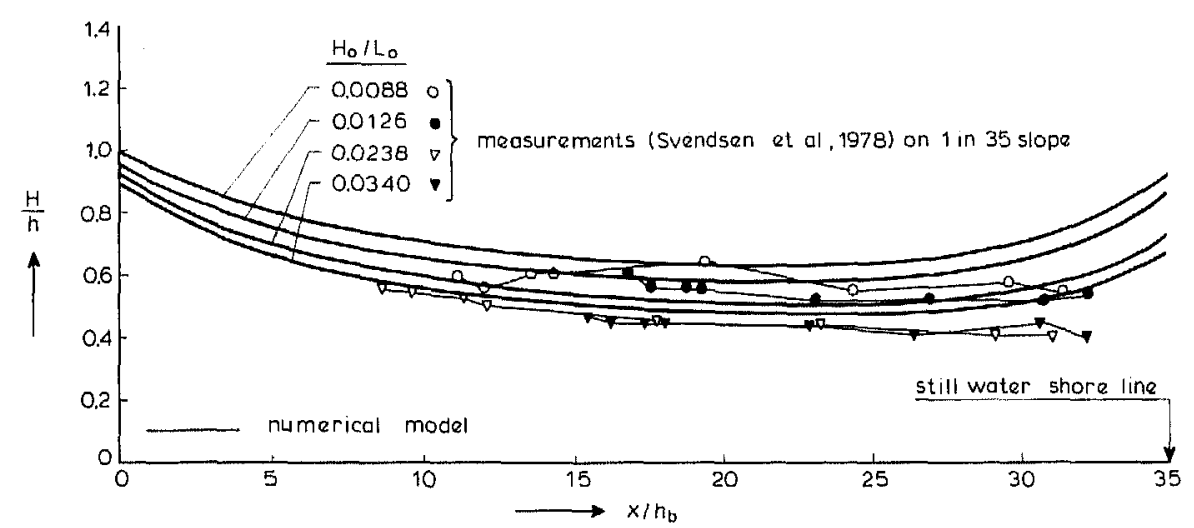

Fig. 15. Comparison between model results and experiments of Svendsen et al. (1978); wave height to mean water depth ratio versus normalized distance to breakpoint.

\section{CONCLUSIONS}

Flow field measurements for two quasi-steady breaking wave conditions on a gently sloping beach confirm the resemblance between quasi-steady breakers, bores, and weak hydraulic jumps. This gives support to a formulation of the dissipation rate of quasi-steady breakers after that of hydraulic jumps.

The periodic version of the classical hydraulic jump formulation is shown to underestimate the measured dissipation rates in the present experiments by 30 to $50 \%$. It is shown that this discrepancy is mainly associated with the turbulent flux of momentum of the flow at the crest section. This effect may be incorporated through a dissipation factor $A_{\epsilon}$. These conclusions are derived for the inner breaking region. 
A simple model is proposed for the wave height decay, based on the solution of a system of differential equations, being simplified forms of the averaged equations of energy and momentum. In the energy equation the dissipation is modelled after that of the hydraulic jump, with the dissipation factor $A_{e}$ constant over the entire surfzone. In the momentum equation the radiation stress is modelled after linear, shallow-water theory with a correction factor $A_{\mathrm{s}}$ which is a constant over the surfzone. For the present experiments the wave height decay and the mean set-up slope appear to be well predicted, if empirically determined values for the factors $A_{\epsilon}$ and $A_{\mathrm{s}}$ are used.

Finally, the model is generalized for a wider range of wave conditions. From an analysis of published data, empirical relations for the model parameters $A_{\epsilon}$ and $A_{\mathrm{s}}$ are derived. Based on the empirical relations the model yields good results for the overall wave height decay and the mean set-up slope for waves of moderate steepness on slopes of 1 in 80 to 1 in 20 . Apparently the less satisfactory results for the initial wave height decay and for the associated qualitative variation of the set-up may be improved by a more sophisticated modelling of the dissipation and radiation stress factors $A_{\epsilon}$ and $A_{s}$ in the initial breaking region.

\section{ACKNOWLEDGEMENT}

This work was done as a part of the Coastal Research Program of the Ministry of Transport and Public Works (Rijkswaterstaat) of the Netherlands. Many thanks are due to Prof. J.A. Battjes for suggesting this study, for valuable comments and for pointing out the analytical solution (5.6). Dr. I.A. Svendsen is thanked for sending additional data required in the comparison of Fig. 15, and for comments on an earlier draft of this paper which resulted in some improvements.

\section{APPENDIX A. EXTRAPOLATION OF THE VELOCITY FIELD}

Velocity measurements were performed in the air-free flow region, i.e. the region roughly below the level of the wave troughs. In the aerated crest region the velocity field was extrapolated as described extensively in Stive and Wind (1982). Here the procedure of extrapolation of the velocities is shortly repeated and evaluated.

The mean horizontal flow profile was extrapolated linearly aided by application of the equation of conservation of mass for a locally steady wave, which reads:

$\int_{-d}^{\zeta} \tilde{u} \mathrm{~d} z=c(\zeta-\bar{\zeta})$

With the propagation speed $c$ and the surface elevation $\xi$ known from the 
measurements, a value for the velocity integral is obtained which serves as a constraint for the extrapolation of the $\bar{u}(z)$ values performed between the highest measurement level and the fluid surface.

The fact that application of the mass conservation equation as described above leaves little margin for different flow results may be confirmed by the results in Fig. a.1. Here the measured and extrapolated horizontal flow results at the crest of the present study are compared with "spilling" breaker measurements of Van Dorn (1978). It is noted that although the extrapolation at the crest is rather drastic in its prediction of a change of trend, a satisfying agreement is found at the higher fluid levels.

In the non-aerated regions of the quasi-steady breaking waves the turbulent velocity intensities $\left(u_{\mathrm{rms}}^{\prime}, w_{\mathrm{rms}}^{\prime}\right)$ behave similar to those in wakes. Therefore, the turbulent intensities were extrapolated on the basis of flow

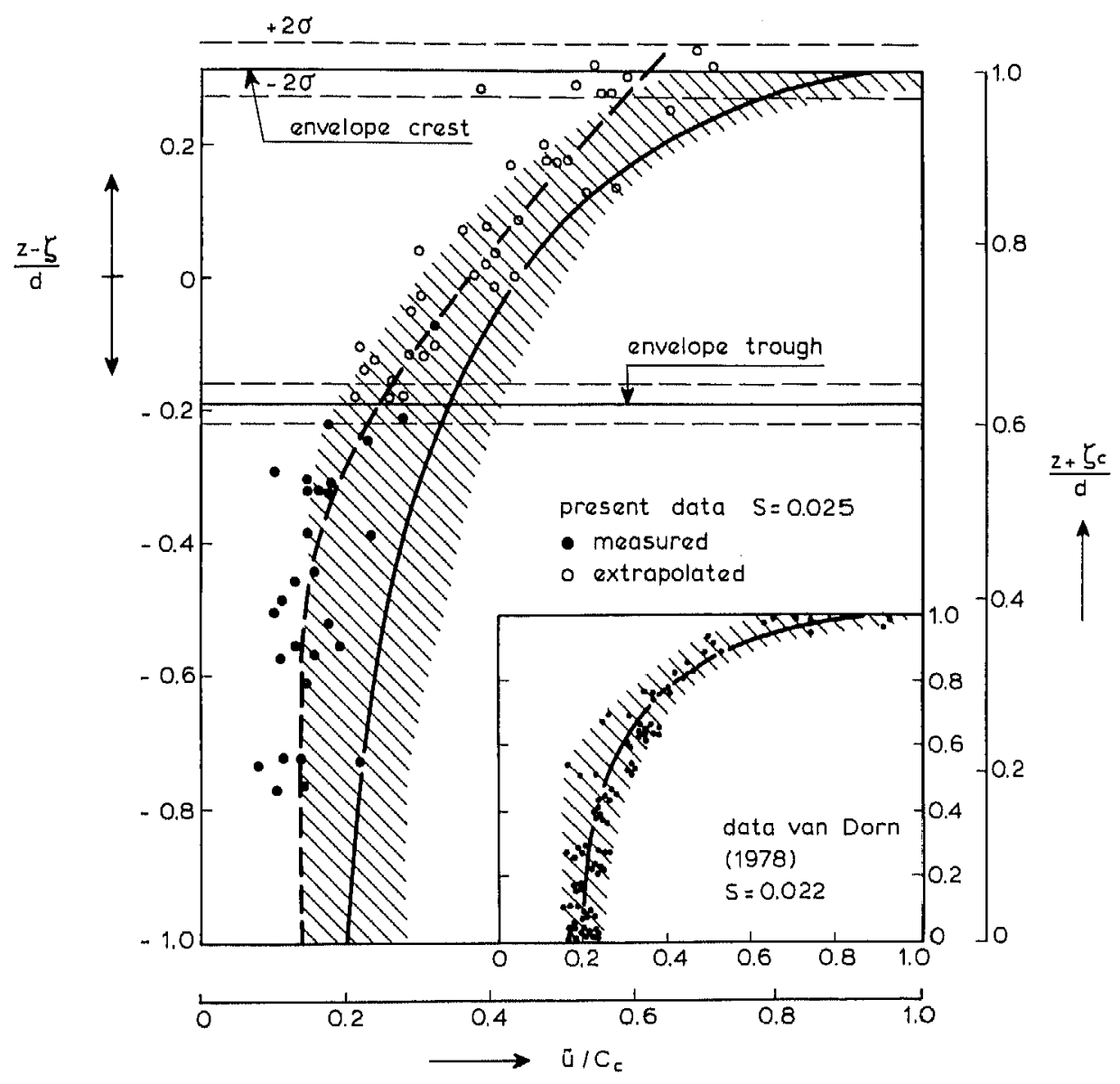

Fig. a.1. Horizontal crest velocities in "spilling" breaking waves. 
results for wakes as basically given by Tennekes and Lumley (1972). Through application of the self preservation hypothesis to the velocity defect, i.e. the difference between the local velocity $\tilde{u}$ and the undisturbed stream velocity $\tilde{u}_{o}$, it is found that the ratio of the turbulent intensities to the local maximum velocity defect, $\tilde{u}_{s}$, behaves like (see also Fig. a.2):

$u_{\mathrm{rms}}^{\prime} / \tilde{u}_{\mathrm{s}}=0.50 \xi^{1 / 2} \exp \left(-1 / 4 \xi^{2}\right)$

$w_{\mathrm{rms}}^{\prime} / \bar{u}_{\mathrm{s}}=0.44 \xi^{1 / 2} \exp \left(-1 / 4 \xi^{2}\right)$

where $\xi$ is a normalized lateral distance to the wake axis which is related to the local velocity defect by:

$\left(\tilde{u}_{\mathrm{o}}-\tilde{u}\right) / \tilde{u}_{\mathrm{s}}=\exp \left(-1 / 2 \xi^{2}\right)$

Both the breaking wave results and the low Froude hydraulic jump results of Rouse et al. (1958) compare reasonably well to these relations (see

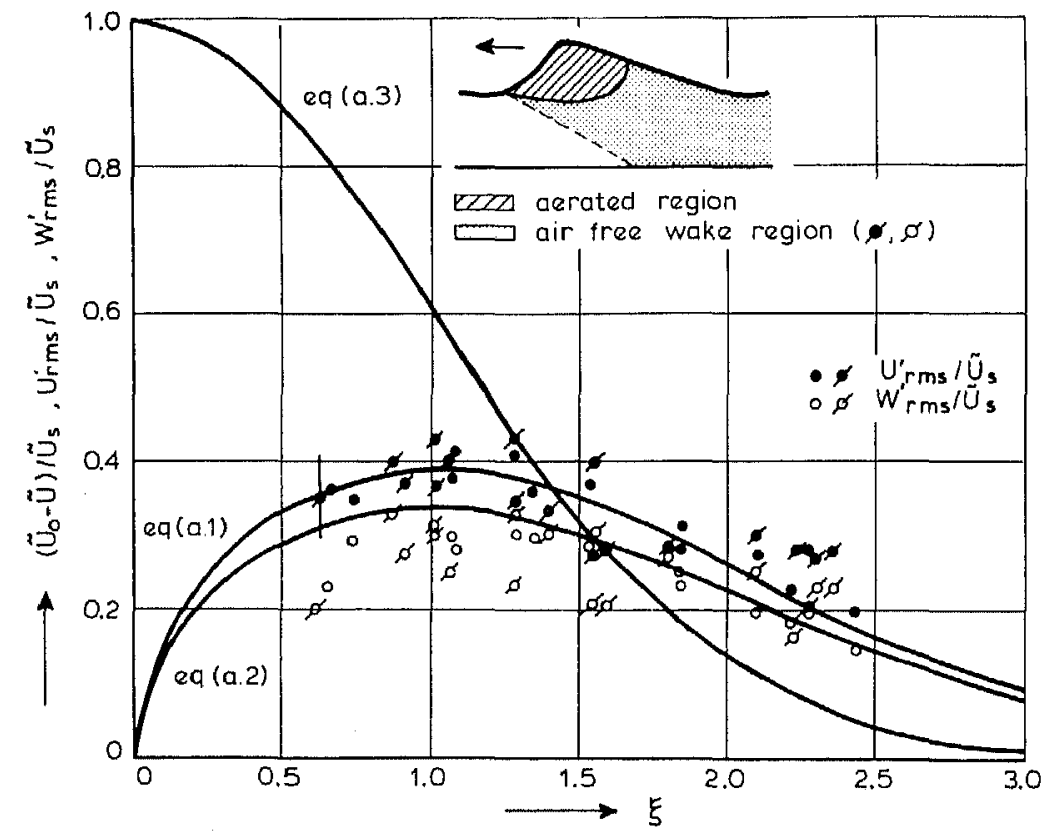

theory for plane wakes

observations for breaking waves inner region

- o. observations hydraulic jump $\left(F_{1}=2\right)$ at $x / d_{2}=2$ and 3

(Rouse et al, 1958)

Fig. a,2, Velocity defect and turbulent intensity in breaking waves and a hydraulic jump. 
Fig. a.2). This confirms the similarity between both classes of flows and it supports the extrapolation procedure which was adopted.

\section{APPENDIX B. HYDRAULIC JUMP DISSIPATION}

Here a concise derivation is given of a "formally corrected" expression for the hydraulic jump dissipation, of which the "classical" expression is shown to be a special case.

Consider a moving control volume in which the ensemble mean motion is steady. The control volume occupies the entire fluid depth. In this reference frame with stationary mean motion, we may distinguish the following ensemble mean (denoted by a tilde) and turbulent (denoted by a prime) quantities:

$(v, w, p, \zeta)=(\bar{v}, \tilde{w}, \bar{p}, \tilde{\zeta})+\left(v^{\prime}, w^{\prime}, p^{\prime}\right)$

in which $v=u-c, \bar{v}=\tilde{u}-c$ and $v^{\prime}=u^{\prime}$, where $u$ is the horizontal component of velocity and $c$ is the wave propagation speed in the frame of reference stationary with respect to the flume (see Fig. 2 for coordinates and notation in the latter frame).

The energy balance for the frame of reference in which the mean motion is steady, neglecting dissipation due to bottom friction, is written as:

$\frac{\partial}{\partial x} \int_{d}\left[p+\rho g z+1 / 2 \rho\left(v^{2}+w^{2}\right)\right] v \mathrm{~d} z=-\epsilon$

where $\epsilon$ is the instantaneous viscous dissipation rate per unit horizontal area.

In order to express the effects of non-uniformity of the mean flow profile, turbulence and non-hydrostatic pressure on the momentum flux and on the energy flux the coefficients as given in Table II are introduced. In the expressions for $\beta^{\text {t }}$ terms considered important, e.g. those representing transport of turbulent stresses, are included whereas terms considered less important, e.g. those representing diffusion of turbulence, are neglected.

Introducing these coefficients in the energy equation and integration from section 1 to 2 yields:

$\left[\rho g V d^{2}+1 / 2 \rho V^{3} d \beta\right]_{2}^{1}=-\epsilon_{\text {corr. }}$.

It is noted that at the boundaries contributions to the fluxes of mass, momentum and energy due to the turbulent fluctuations in $\zeta$ are assumed negligible. Furthermore, turbulent interactions between $p$ and $v$ are assumed negligible in (the derivation of) this equation. Using the continuity equation $(V d=-c h)$ and after some rearrangement the following expression may be derived for the viscous dissipation of energy in the volume 
bounded by sections 1 and 2 :

$\epsilon_{\text {corr. }}=\rho g c h\left(d_{1}-d_{2}\right)+1 / 2 \rho c^{3} h^{3}\left(\frac{\beta_{1}}{d_{1}^{2}}-\frac{\beta_{2}}{d_{2}^{2}}\right)$

Under the assumptions of uniform velocity, absence of turbulence and hydrostatic pressure in sections 1 and 2 , implying $\beta^{\mathrm{v}}=1, \beta^{\mathrm{t}}=0$ and $\beta^{\mathrm{p}}=0$, the following expression for the hydraulic jump or bore dissipation results:

$\epsilon_{\text {bore }}=\rho g c_{\text {bore }} h\left(d_{2}-d_{1}\right)^{3} /\left(4 d_{1} d_{2}\right)$

where $c_{\text {bore }}=\left(1 / 2 g d_{1} d_{2}\left(d_{1}+d_{2}\right) h^{-2}\right)^{1 / 2}$.

which transforms into the classical expression for a hydraulic jump using the continuity equation and $h=d_{1}$. Alternatively the effects of non-uniformity, turbulence and non-hydrostatic pressure on the bore dissipation may now be expressed by:

$\epsilon_{\text {corr, }}=\epsilon_{\text {bore }} A_{\mathrm{c}}^{1 / 2}\left[1+\frac{(\delta+1)^{2}}{(\delta-1)^{2}}\left(A_{\mathrm{c}} A_{\mathrm{B}}-1\right)\right]$

where $A_{\mathrm{B}}=\frac{\delta^{2} \beta_{1}-\beta_{2}}{\delta^{2}-1}, A_{\mathrm{c}}=\left(\frac{c}{c_{\text {bore }}}\right)^{2}=\frac{\delta-1}{\alpha_{1} \delta-\alpha_{2}}$ and $\delta=d_{2} / d_{1}$.

The above derivation and resulting expression (b.1) for $A_{\epsilon}$ closely correspond to Svendsen et al. (1978). The difference between their equation (55) and expression (b.1) lies in the presence of the term $A_{\mathbf{c}}^{1 / 2}$, the inclusion of the pressure effects in $\alpha$ and $\beta$ and the inclusion of the turbulent effects in $\beta$.

\section{REFERENCES}

Battjes, J.A., 1974. Computation of set-up, longshore currents, run-up and overtopping due to wind-generated waves. Comm. on Hydraulics, Dept. of Civil Eng., Delft Univ. of Technology, 74-2, $244 \mathrm{pp}$.

Cokelet, E.D., 1977. Steep gravity waves in water of arbitrary uniform depth. Philos. Trans. R. Soc. London, Ser. A, 286: 183-230.

Divoky, D., Le Méhauté, B. and Lin, A., 1970. Breaking waves on gentle slopes. J. Geophys. Res., 75: 1681-1692

Horikawa, K. and Kuo, C-T, 1966. A study on wave transformation inside the surf zone. Proc. 10th Coastal. Eng. Conf, Tokyo, pp. 217-233.

Hwang, L. and Divoky, D., 1970. Breaking wave set-up and decay on gentle slopes. Proc. 12th Coastal. Eng. Conf., pp. 377-389.

LeMehhauté, B., 1962. On the nonsaturated breaker theory and the wave run-up. Proc. 8th Coastal. Eng. Conf., pp. 77-92.

Rouse, H., Siao, T.T. and Nagarathman, S., 1958. Turbulence characteristics of the hydraulic jump. Proc. A.S.C.E., 84(HY1): 1-30. 
Singamsetti, S.R. and Wind, H.G., 1980. Characteristics of shoaling and breaking periodic waves normally incident to plane beaches of constant slope. Delft Hydraulics Laboratory, Report M 1371.

Stive, M.J.F., 1980. Velocity and pressure field of spilling breakers. Proc. 17th Coastal Eng. Conf., Sydney, pp. 547-566.

Stive, M.J.F., 1983. Two-dimensional breaking of waves on a beach. Pressure field in waves shoaling and breaking on a plane beach. Delft Hydraulics Laboratory, Report M 1585, Part II.

Stive, M.J.F. and Wind, H.G., 1982. A study of radiation stress and set-up in the nearshore region. Coastal Eng., 6: 1-25.

Svendsen, I.A. and Madsen, P.A., 1981. Energy dissipation in hydraulic jumps and breaking waves. Institute of Hydrodynamics and Hydraulic Eng., Techn. Univ. of Denmark, Progress Report no. 55, pp. 39-47.

Svendsen, I.A., Madsen, P.A, and Buhr Hansen, J., 1978. Wave characteristics in the surfzone. Proc. 16th Coastal Eng. Conf., Hamburg, pp. 520-539.

Tennekes, H. and Lumley, J.L., 1972. A First Course in Turbulence. MIT Press, Cambridge, Mass., $300 \mathrm{pp}$.

Van Dorn, W.G., 1976. Set-up and run-up in shoaling breakers. Proc. 15th Coastal Eng. Conf., Hawaii, pp. 738-751. 